\title{
The Flow-Heat Coupling Study of Cooling Circulating Channel of Magnetic Drive Pump
}

\author{
Zhenjun GAO*, Jianbo ZHANG**, Wenyang LI***, Jintao LIU****, Changqing SI*****, \\ Feng HONG******, Jianrui LIU*******, Xiaotao SHI********* \\ *College of Mechanical and Power Engineering/Engineering Research Center of Eco-environment in Three Gorges Res- \\ ervoir Region, Ministry of Education, China Three Gorges University, Yichang 443002, China; \\ E-mail:570186276@qq.com \\ **College of Mechanical and Power Engineering, China Three Gorges University, Yichang 443002, China, \\ E-mail:1053300810@qq.com \\ ***College of Mechanical and Power Engineering, China Three Gorges University, Yichang 443002, China, \\ E-mail:1012377178@qq.com \\ ****Beijing Institute of Control Engineering, Beijing 100190, China, E-mail: liujintao86@hotmail.com \\ *****College of Mechanical and Power Engineering, China Three Gorges University, Yichang 443002, China, \\ E-mail: $1061070924 @ q q . c o m$ \\ ******College of Mechanical and Power Engineering in Three Gorges Reservoir Region, China Three Gorges \\ University, Yichang 443002, China; E-mail: yaphet_ft@163.com \\ *******Research Center of Fluid Machinery Engineering and Technology, Jiangsu University, Zhenjiang 212013, China, \\ E-mail:1787292285@qq.com \\ ********Engineering Research Center of Eco-environment in Three Gorges Reservoir Region, Ministry of Education, \\ China Three Gorges University, Yichang, 443002, China, E-mail: sxtshanghai@163.com (Corresponding author) \\ crossref http://dx.doi.org/10.5755/j02.mech.29651
}

\section{Introduction}

Magnetic driven centrifugal pump is a leak-free pump [1-2], mainly used for conveying strong corrosion, toxic, flammable and valuable medium in the process of industrial production. It is widely used in chemical industry, metallurgy, light industry, food, aerospace, aviation and other industries in recent years because of its characteristics of full seal and no leakage. During the rotation of the inner and outer magnetic rotors of the magnetic drive centrifugal pump, Metal isolation sleeve will generate magnetic eddy current heat; If the heat cannot be cooled in time, the inner and outer magnetic rotors will be demagnetized at high temperature, and eventually the magnetic drive centrifugal pump will fail. After the magnetic eddy current heat is generated, a part of the medium needs to be introduced from the conveyed medium to take the heat away, this part will cause the loss of cooling flow, and the efficiency of magnetic drive centrifugal pump is lower than that of ordinary centrifugal pump. Many scholars [3-7] have conducted many studies on the cooling loop of the magnetic driven centrifugal pump. Kong fanyu et al. [8-10] simplified the structure of the cooling circulation loop of the pump, and used mathematical methods to analyse the flow resistance coefficient of each element inside the pump. At present, the research of magnetic driven centrifugal pump cooling loop is mainly focused on the internal circulation flow and circulation loop structure, the internal heat flow coupling lack of in-depth research.

Convective heat transfer refers to the process of convective exchange of heat between fluids flowing through objects at different temperatures, mainly including forced convection, natural convection and conjugate heat transfer. The research of magnetic drive pump cooling circulating channel involved in this paper belongs to the category of forced convection heat transfer. The medium in the cooling circulating channel flows through the surface of the isolation sleeve under the action of the pressure difference between the two ends. The surface temperature of the isolation sleeve is relatively high. Convection heat transfer occurs between the medium and the surface of the isolation sleeve, and the heat in the isolation sleeve is absorbed by the medium. Convection heat transfer can be solved by numerical calculation. The solution process [11-13] is as follows: 1) Conduct model and analysis for each physical field. 2) Define boundary conditions for the region to solve. The boundary conditions of convection heat transfer problems mainly include temperature continuity, heat flux continuity and the gradual relationship between temperature and heat flow density. In the process of magnetic drive pump operation, the wall of the isolation sleeve is a constant heat flux output surface, which belongs to the second boundary condition. 3) On the basis of the above, the convective heat transfer region is solved by the calculation method of "Partition to solve and boundary coupling" until the convergence is completed.

\section{The method of numerical calculation}

\subsection{Pump structure}

The structure of magnetic drive centrifugal pump is mainly composed of $1-11$ parts, as shown in Fig. 1 . The working principle of the magnetic drive centrifugal pump is that the driving machine drives the outer magnetic rotor of the pump to rotate, and the outer magnetic rotor is connected to the inner magnetic rotor through the action of a magnetic field and drives the impeller to rotate. When the pump is filled with medium, the impeller rotates at a high speed under the drive of the driver. During the rotation, the pump inlet sucks in the medium, and the pump outlet ejects the medium. 


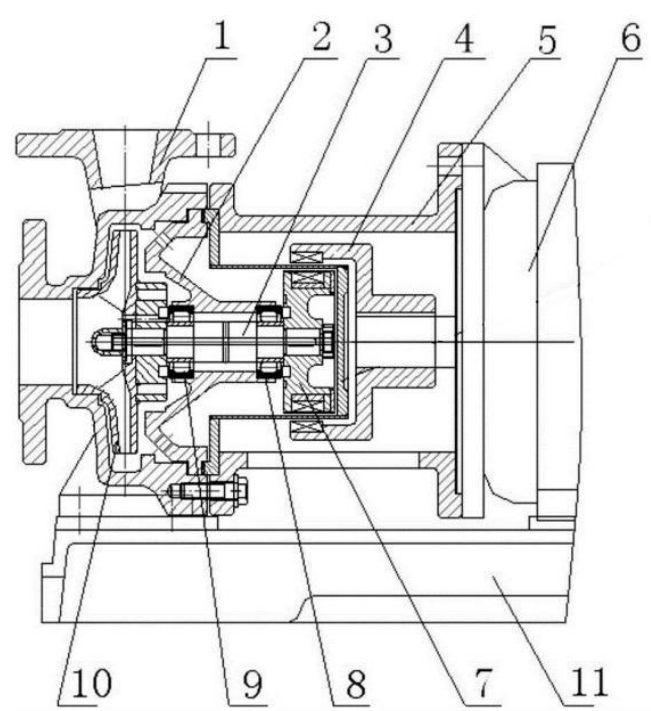

Fig. 1 Magnetic drive centrifugal pump structure diagram:

1 - pump body; 2 - pump cover; 3 - pump shaft;

4 - outer magnetic rotor; 5 - connection frame;

6 - driving machine; 7 - inner magnetic rotor;

8 - thrust plate; 9 - bearing; 10 - impeller;

11 - bracket

The flow of medium in the cooling circulation channel of the magnetically driven centrifugal pump is shown in Fig. 2. Part of the medium enters the isolation sleeve through the return hole, and flows out through the bearing gap and the inner shaft middle hole. The above process can take away part of the heat, and at the same time can play a role in lubricating the bearing.

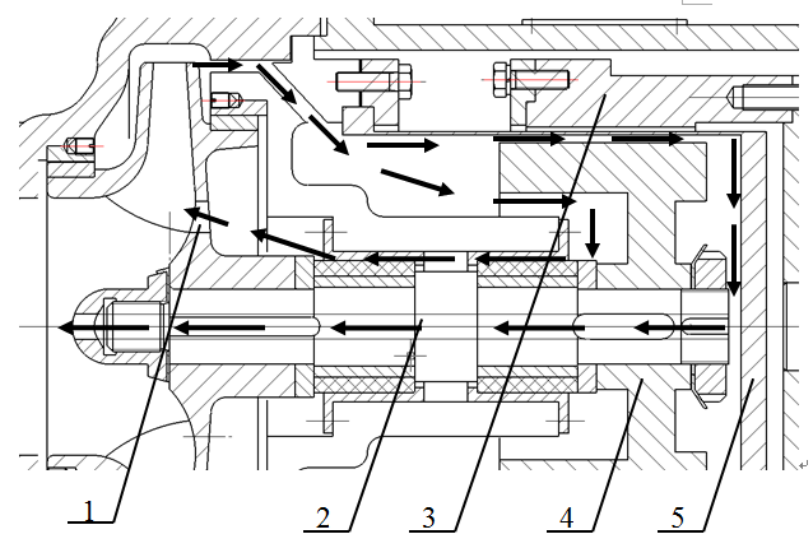

Fig. 2 Schematic diagram of internal circulation structure: 1 - balance hole; 2 - recirculation hole; 3 - outer magnetic rotor; 4 - Inner magnetic rotor; 5 - isolation sleeve

\subsection{The calculation model and mesh generation}

In this study, all the flow areas inside the magnetically driven centrifugal pump are taken as the calculation domain. ICEM software is used to mesh the overall model of the magnetically driven centrifugal pump, and the formed mesh is dominated by a hexahedron. Since the flow-heat coupling of the cooling circuit of the magnetically driven centrifugal pump is selected as the main analysis object in this paper, the grid encryption processing is used for the cooling circulation channel and the part with large fluid flow changes to improve the calculation accuracy.
After verification of grid independence, the total number of computational grids of the magnetically driven centrifugal pump is determined to be 5618310 . And the calculation area model of the magnetic drive centrifugal pump is shown in Fig. 3. In addition, the calculation of this model involves more small-size clearance flows. SST $k$ - $\omega$ model has both the reliability of $k-\omega$ model in the calculation of viscous flow near the wall region and the accuracy of $k-\varepsilon$ model in the calculation of unrestricted flow in far field. SST $k$ - $\omega$ model has obvious advantages in the calculation of small-size clearance flows, so the SST $k$ - $\omega$ model was adopted in this paper.

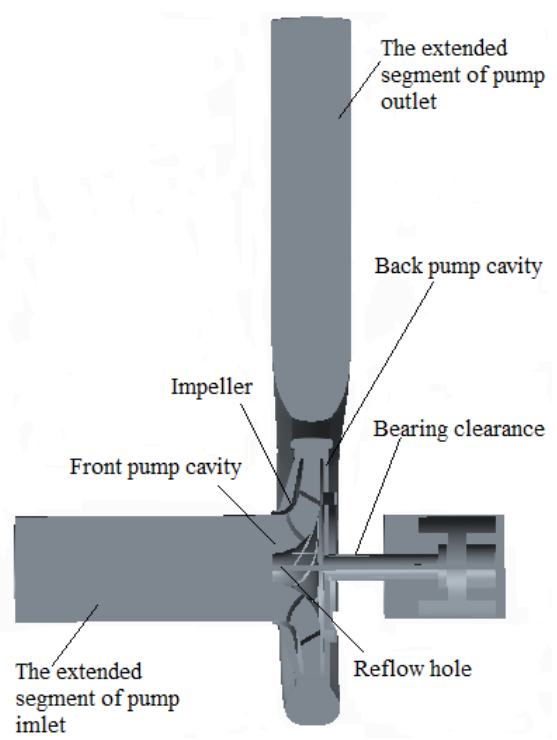

a

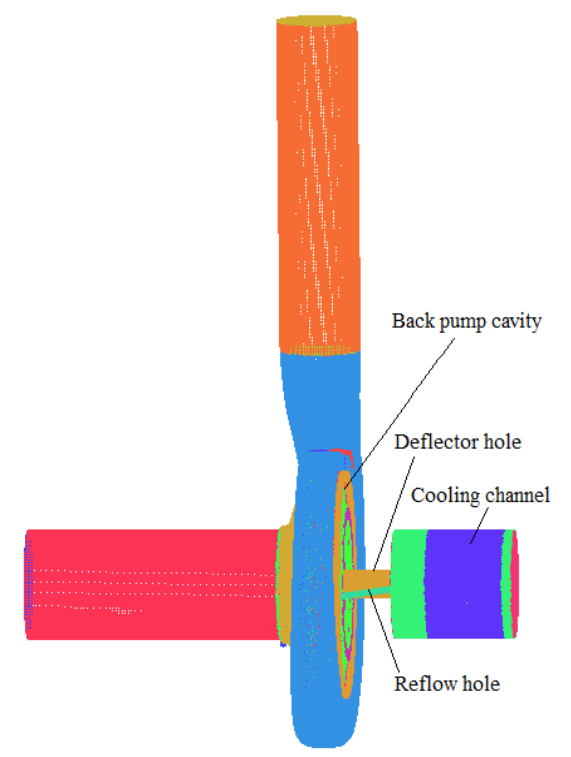

b

Fig. 3Magnetic drive centrifugal pump calculation area model: a - Magnetic drive centrifugal pump calculation area assembly drawing; $b$ - Magnetically driven centrifugal pump calculation area grid

\subsection{Calculation of heat source}

The power loss of magnetic drive pump mainly includes hydraulic loss, volume loss, mechanical loss, mag- 
netic eddy current loss, internal and external magnetic rotor friction loss, etc. The heat source in the cooling circulating channel mainly considers the heat generated by the eddy current loss. It is assumed that the wall of the isolation sleeve generates uniform heat, and the eddy current loss value of the magnetic drive coupling of the centrifugal pump under a certain working condition is constant and the heat is constant. Under different working conditions, the flow head of the magnetic drive pump is different, and the load on the input end of the pump shaft of the magnetic drive coupling is different, resulting in the magnetic rotation Angle, magnetic torque, magnetic eddy current loss and transfer efficiency of the magnetic drive coupling are different. The eddy current loss under different working conditions can be calculated according to formulas (1) and (2) [14-16]:

$$
\begin{aligned}
& T_{K}=T_{\text {max }} \sin \left(\frac{m \Phi}{2}\right) \\
& T_{\omega}=T_{\omega \max } \cos \left(\frac{m \Phi}{4}\right)
\end{aligned}
$$

Precise calculation of the magnetic eddy current loss under different working conditions, should put the measured magnetic drive coupling power converted into static magnetic torque $T_{k}$, according to the formula (1) to calculate the corresponding Magnetic angle $\varphi$, then Accord- ing to the magnetic rotation angle test, the magnetic eddy current torque $T_{w}$ is obtained, $T_{w \max }$ is obtained by the formula (2), then the value of magnetic eddy current loss corresponding to any Magnetic angle can be calculated. Based on the numerical calculation results of the external characteristics of the magnetic drive pump, the pump shaft power value under different working conditions was extracted, and the pump shaft torque value was calculated and obtained. The magnetic rotation Angle corresponding to the magnetic drive coupling under different working conditions was calculated according to the formula (1). Based on the test results of eddy current loss of magnetic coupling, the maximum eddy current torque $T_{\text {wmax }}$ was calculated according to formula (2), and then the eddy current loss corresponding to magnetic drive coupling under different working conditions was calculated according to different magnetic rotation angles. Finally, the transfer efficiency of magnetic drive coupling under different working conditions is predicted by referring to the statistical data of loss in the case of no isolation sleeve. The data used to calculate the corresponding magnetic rotation angle under different working conditions is the pump shaft torque, so the eddy current loss value obtained is relatively large, but within the allowable range. The eddy current loss and transfer efficiency of magnetic drive coupling under different working conditions are shown in Table 1.

Table 1

Eddy current loss and transfer efficiency of magnetic drive coupling under different working conditions

\begin{tabular}{|l|l|l|l|l|l|l|l|l|}
\hline Flow & $0.2 \mathrm{Q}$ & $0.4 \mathrm{Q}$ & $0.6 \mathrm{Q}$ & $0.8 \mathrm{Q}$ & $1.0 \mathrm{Q}$ & $1.2 \mathrm{Q}$ & $1.4 \mathrm{Q}$ & $1.6 \mathrm{Q}$ \\
\hline Pump shaft power, $\mathrm{kW}$ & 15 & 18.5 & 23.8 & 28 & 31.5 & 34.5 & 37 & 40 \\
\hline Pump shaft torque, Nm & 98.8 & 121.8 & 156.7 & 184.4 & 207.5 & 227.2 & 243.7 & 263.4 \\
\hline Eddy current loss, W & 2262 & 2250 & 2225 & 2199 & 2173 & 2145 & 2119 & 2083 \\
\hline Transfer efficiency, \% & 83.2 & 85.9 & 88.8 & 90.4 & 91.4 & 92.2 & 92.7 & 93.3 \\
\hline
\end{tabular}

As can be seen from Table 1, different operating conditions have less impact on magnetic eddy current loss and greater impact on transfer efficiency. Under the condition of small flow rate, the magnetic eddy current loss is relatively large. As the flow rate increases, the magnetic eddy current loss decreases gradually. Mainly because of the small flow conditions, the pump shaft power and pump shaft torque value are small, the corresponding Magnetic Angle is small, the Angle of magnetic force line between internal and external magnetic rotor and isolation sleeve becomes larger. Under the same rotating speed, the magnetic flux passing through the isolation sleeve in unit time increases, and the induced electromotive force increases, resulting in a large loss of magnetic eddy current. With the increase in flow rate, the magnetic rotation Angle between internal and external magnetic rotors increases, and the average magnetic induction intensity acting on the isolation sleeve decreases, resulting in the decrease of magnetic eddy current loss. Under the condition of small flow rate, the transfer efficiency of magnetic drive coupling is small. With the increase in flow rate, the pump shaft power and pump shaft torque increase, and the magnetic eddy current loss does not change much, so the transfer efficiency increases.

In conclusion, in the design process of magnetic drive coupling, attention should be paid to the design of magnetic rotation Angle corresponding to rated torque. The magnetic rotation Angle is too small and the eddy current loss is relatively large. If the magnetic rotation Angle is too large, the difference between the rated torque value and the maxi-mum magnetic torque value is relatively small. In the case of starting and overload, magnetic drive coupling is prone to slip, which affects the transfer of torque. The design of magnetic rotation Angle corresponding to rated torque is mainly related to the selection of overload multiple of maximum magneto static torque. If the overload multiple is too large, the maximum magneto static torque value will increase, the number of permanent magnetic materials will increase, the magnetic rotation Angle corresponding to the rated torque will be small, and the transfer efficiency will be relatively low. The overload multiple is too small, the maximum magneto static torque value is small, the magnetic rotation Angle corresponding to the rated torque is large, and the transfer efficiency is high, but the internal and external magnetic rotor are prone to slip-off. After the magnetic eddy current loss is determined under different working conditions, the value of magnetic eddy current loss is converted into heat flux and loaded onto the inner wall of the isolation sleeve during numerical calculation (the heat source surface shall be set in advance during pre-treatment).

\subsection{Calculation settings}

A part of the heat is transmitted and radiated from the outer surface of the isolation sleeve, and its value is 
small, so it can be considered that eddy current heat is cooled entirely by medium in engineering applications. The calculation of heat generated by sliding bearing and friction loss of internal magnetic rotor in the medium is complex and difficult to accurately solve. Therefore, this paper only considers the case when eccentricity is zero and rotor weight is not considered in the research process. In this case, the heat value generated by friction of sliding bearing is smaller than that generated by eddy current heating. In order to simplify the calculation process and improve the calculation efficiency, only eddy current heat inside the isolation sleeve is considered in the numerical calculation in this paper, and the inner surface of the isolation sleeve is set as the heat source surface. In the process of numerical calculation, the inlet boundary condition is set as the flow inlet. The outlet boundary condition is set as the pressure outlet. Solid wall surface is set as non-sliding wall surface, and automatic wall function method is used near the wall surface. The water body in the impeller channel is set as rotating body, and the water body in the pressurized water chamber, bearing clearance, cooling circulating channel and front and rear pump cavity is non-rotating body. When the wall condition is set, the wall in contact with the rotating part of the water body is set as the rotating wall, the rotation speed is the pump speed, and the wall surface in contact with the fixed flow part is set as the stationary wall. The interface between rotating water body and non-rotating water body is set as the interface between dynamic and static of type Frozen Rotor. This type of interface plays an important role in the dynamic and static coupling between two parts of water bodies, and the GGI method of CFX software is adopted for the connection between the interface grids [17-18]. The initial medium temperature is set as normal temperature $(298 \mathrm{~K})$, the inner wall of the isolation sleeve (heat source surface) is set as constant temperature wall condition, other walls are set as adiabatic wall surface, and the convergence precision is set as $10^{-4}$.

\section{Analysis of numerical results}

\subsection{Distribution of temperature characteristics in cooling circulating channel}

\subsubsection{Temperature distribution in cooling circulating chan-} nel under different working conditions

The temperature distribution in the cooling circulating channel under different working conditions is shown in Fig. 4. As can be seen from Fig. 4, the medium in the cooling circulating channel carries away the eddy current heat cooling generated in the isolation sleeve through the gap of the isolation sleeve. The cooling medium with highertemperature flows into the low-pressure area of impeller through the reflow hole, and the medium velocity in the low- pressure area of the impeller inlet through the reflux port is large, and the impeller inlet flow state will deteriorate, if it collides with the medium in the direction of pump inlet. On the other hand, the medium temperature flowing out of the reflow hole is relatively high, and cavitation is easy to occur at the impeller inlet under the same working condition, thus reducing the cavitation performance of the pump. In addition, it can be seen from Fig. 3, the highest temperature in the cooling circulating channel appears at the position where the gap of the isolation sleeve is close to the bottom of the isolation sleeve, and the medium temperature at the bottom of the isolation sleeve and the reflow hole is lower.

The changes in temperature rise and cooling flow rate in the cooling circulating channel under different working conditions are shown in Table 2. As can be seen from Table 2, the minimum temperature change in the cooling circulation channel is small under different working conditions, and the maximum temperature changes with the flow rate. The maximum temperature variation range in the cooling circulating channel under different working conditions is very small, and it can be basically considered that the temperature rise in the cooling circulating channel is the same, which is mainly because the eddy current loss and the cooling cycle have the same trend with the flow rate, and the eddy current loss and the cooling circulating flow are large under the low-flow working condition.

As the flow rate increases, the eddy current loss and cooling circulation flow decrease, and the heat generated by the eddy current loss is mainly cooled by the cooling circulation medium. Therefore, the changes in the corresponding cooling circulation flow exactly offset the changes in temperature rise caused by the changes in the eddy current loss, resulting in basically no significant changes in the temperature rise under different working conditions. In addition, it can be seen from Table 2 that under the condition of $0.4 Q$, the cooling flow rate flowing through the gap of the isolation sleeve in the cooling passage is $0.393 \mathrm{~kg} / \mathrm{s}$. With the increase in the flow rate, the cooling flow rate in the cooling circulating channel decreases gradually, which is mainly because the size of the cooling circulation flow rate in the cooling circulating channel is mainly affected by the pressure difference in the cooling circulating channel. The greater the pressure difference, the greater the cooling circulation flow rate. When the flow rate increases, the pressure value in the flow passage of the pressurized water chamber decreases, the pressure value near the inlet of the deflector hole in the cooling circulating channel decreases, and the pressure difference in the cooling circulating channel decreases, so the cooling circulation flow decreases. According to the comprehensive analysis, the temperature rise in the cooling circulating channel meets the design requirements, and the design and calculation of the cooling circulation channel have certain reliability.

Table 2

The changes of temperature rise and cooling flow rate in the cooling circulating channel under different working conditions

\begin{tabular}{|c|c|c|c|c|c|c|}
\hline Working condition & $0.4 Q$ & $0.6 Q$ & $0.8 Q$ & $1.0 Q$ & $1.2 Q$ & $1.4 Q$ \\
\hline The lowest temperature, $\mathrm{K}$ & 298.047 & 298.048 & 298.035 & 298.030 & 298.032 & 298.030 \\
\hline The highest temperature, $\mathrm{K}$ & 301.162 & 300.985 & 301.105 & 301.023 & 301.029 & 300.999 \\
\hline Cooling flow rate within isolation sleeve clearance, $\mathrm{kg} / \mathrm{s}$ & 0.393 & 0.378 & 0.365 & 0.354 & 0.340 & 0.323 \\
\hline
\end{tabular}


Temperature
Contour 1
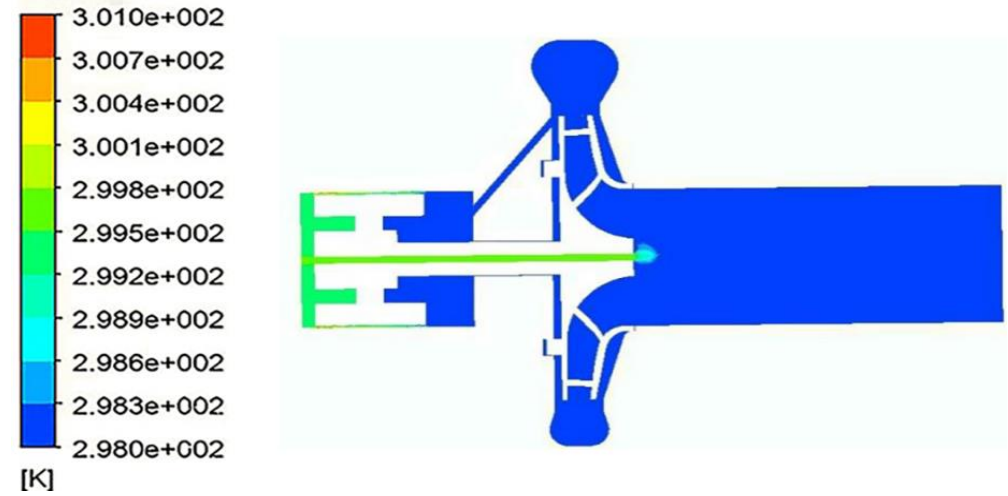

a

Temperature

Contour 1

T.010e+002

$3.007 e+002$

$3.004 e+002$

$3.001 e+002$

$2.998 \mathrm{e}+002$

$2.995 e+002$

$2.992 \mathrm{e}+002$

$2.989 e+002$

$2.986 e+002$

$2.983 e+002$

$2.980 \mathrm{e}+\mathrm{CO} 2$

[K]

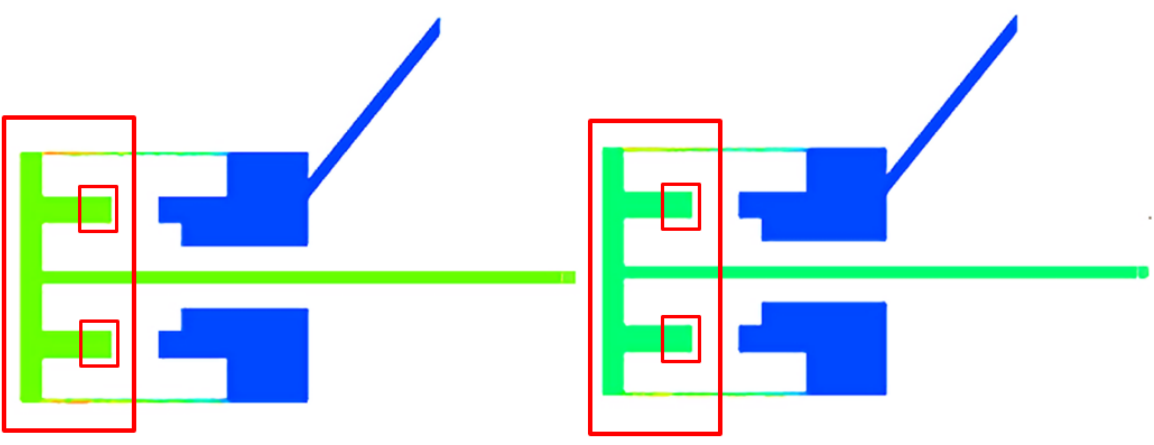

b

c

Temperature Contour 1

\begin{tabular}{ll}
\hline $3.010 e+002$ \\
$3.007 e+002$ \\
$3.004 e+002$ \\
$3.001 e+002$ \\
$2.998 e+002$ \\
$2.995 e+002$ \\
$2.992 e+002$ \\
$2.989 e+002$ \\
$2.986 e+002$ \\
$2.983 e+002$ \\
$2.980 e+002$
\end{tabular}
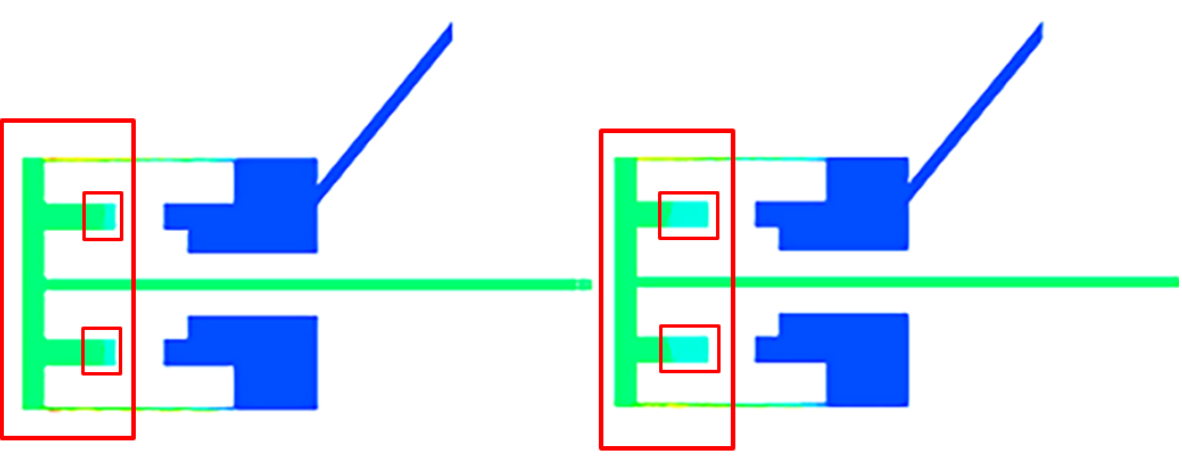

d

e

Temperature

\begin{tabular}{|c|}
\hline \multirow[b]{2}{*}{$3.007 e+002$} \\
\hline \\
\hline $3.004 e+002$ \\
\hline $3.001 \mathrm{e}+002$ \\
\hline $2.998 e+002$ \\
\hline $2.995 e+002$ \\
\hline $2.992 \mathrm{e}+002$ \\
\hline $2.989 e+002$ \\
\hline $2.986 e+002$ \\
\hline $2.983 e+002$ \\
\hline $2.980 \mathrm{e}+\mathrm{CO} 2$ \\
\hline
\end{tabular}

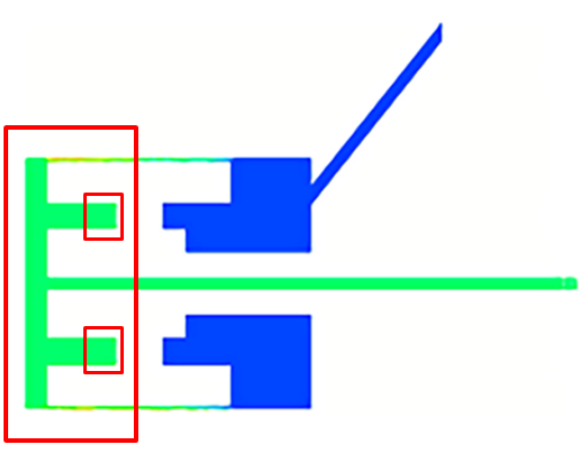

$\mathrm{f}$

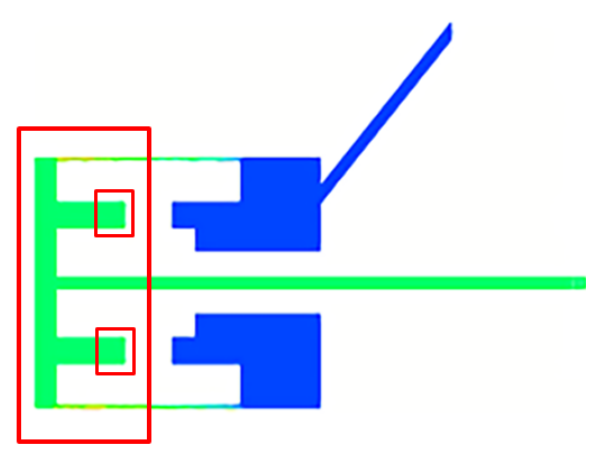

g

Fig. 4 Temperature distribution in cooling circulating channel under different working conditions: a) Overall structure diagram; b) $0.4 Q ;$ c) $0.6 Q ;$ d) $0.8 Q ;$ e) $1.0 Q ;$ f) $1.2 Q ;$ g) $1.4 Q$ 
3. 1. 2. Temperature and pressure distribution in the gap of isolation sleeve

The temperature and pressure distribution in the gap of the isolation sleeve under the design condition is shown in Fig. 5.

As can be seen from Fig. 5, the temperature in the gap of the isolation sleeve gradually increases along the gap of the isolation sleeve toward the bottom, and the highest temperature area appears near the bottom of the isolation sleeve and close to the wall surface (A). At the same longitudinal section, the temperature close to the wall area of the isolation sleeve is higher than that far from the wall, which is mainly related to the convection heat transfer of the medium. The pressure in the gap of the isolation sleeve decreases gradually along the direction of the isolation sleeve toward the bottom, and the lowest pressure area appears near the bottom of the isolation sleeve and away from the wall surface (A), which is mainly related to the pressure drop loss in the gap of the isolation sleeve. At the same longitudinal section, the pressure near the wall area of the isolation sleeve is higher than that far away from the wall area. According to the cavitation formation mechanism, under the same pressure condition, the higher the temperature, the more likely cavitation will occur. Under the same temperature condition, the lower the pressure, the more likely cavitation will occur. Therefore, the above (A) area is the area where cavitation is most likely to occur in the gap of the isolation sleeve, where cavitation is most likely to occur in the middle of the gap of the isolation sleeve near the bottom (near the region with high temperature).

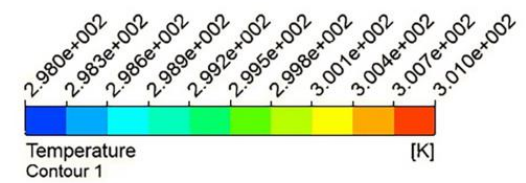

A
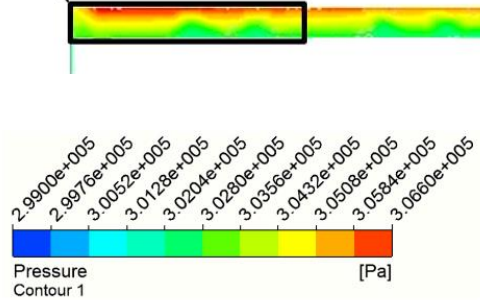

Fig. 5 Temperature and pressure distribution in isolation sleeve clearance under design conditions: a - Temperature; b - Pressure

\section{1. 3. Temperature and pressure distribution at the bottom} of the isolation sleeve

The temperature and pressure distribution of the bottom section of the isolation sleeve under the design condition is shown in Fig. 6. As can be seen from Fig. 6, the middle position at the bottom of the isolation sleeve has the lowest pressure and the highest temperature. The higher
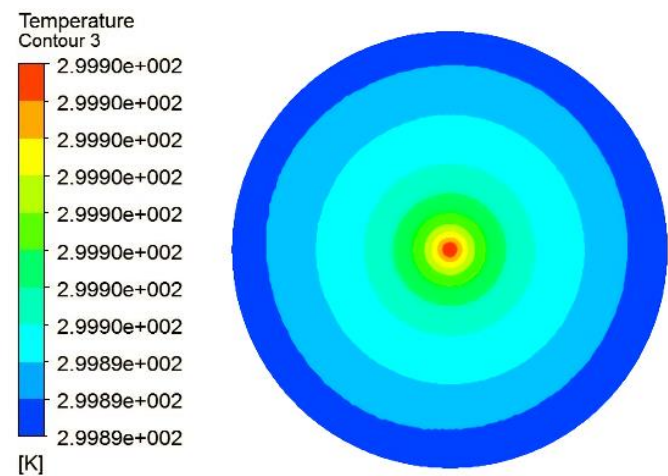

a temperature part of the bottom of the isolation sleeve overlaps the lower pressure part of the bottom of the isolation sleeve, and the coincidence area is located just near the centre of the bottom of the isolation sleeve. According to the cavitation mechanism mentioned in Fig. 4, the risk of cavitation at the middle position at the bottom of the isolation sleeve is the highest.
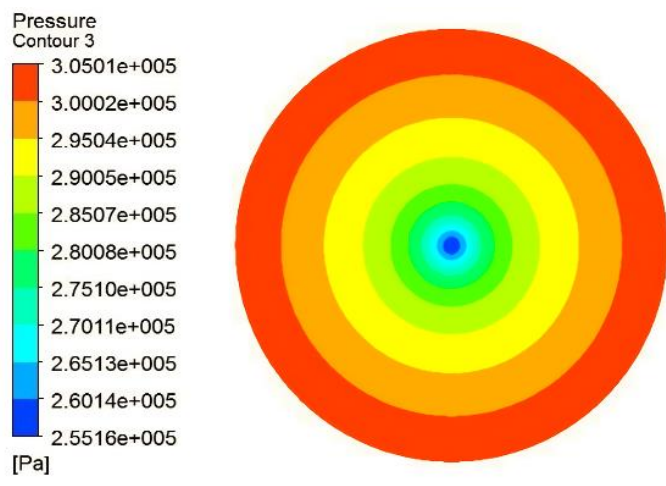

b

Fig. 6 The temperature and pressure distribution of the bottom section of the isolation sleeve under the design condition:

a) Temperature; b) Pressure 
The temperature and pressure distribution in the reflow hole under the design condition is shown in Fig. 7. As can be seen from Fig. 7a, the temperature at the inlet of the reflow hole is relatively high, and the temperature inside the reflow hole gradually decreases along the exit direction of the reflow hole. As can be seen from Fig. 7b, the pressure at the inlet of the reflow hole is large, and the pressure gradually decreases along the exit direction of the reflow hole.

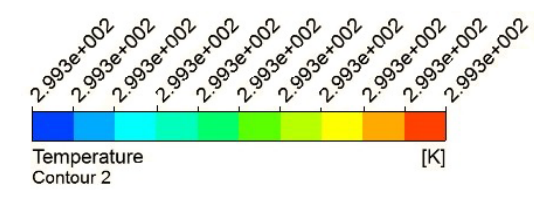

The pressure in the area near the exit of the reflow hole is the lowest. As can be seen from Fig. 4, the temperature variation amplitude in the reflow hole is small, so cavitation is more likely to occur in the area near the outlet of the reflow hole. In addition, the temperature and pressure at the inlet of the reflow hole appear irregular distribution, which is caused by the sudden contraction of the medium flow state at the inlet of the reflow hole.

b

Fig. 7 The temperature and pressure distribution in the reflow hole under the design condition: a) Temperature; b) Pressure

\subsection{Distribution of convective heat transfer}

3. 2. 1. The distribution of convection heat transfer coefficient

The convection heat transfer coefficient is a physical quantity that measures the heat transfer capacity between the fluid and the solid surface. Specifically, the heat transfer coefficient is defined as the heat transferred per second by the wall area of $1 \mathrm{~m} \times 1 \mathrm{~m}$ when the temperature difference between the fluid and the solid surface is $1 \mathrm{~K}$. There are many factors influencing convective heat transfer, including the physical properties of fluid, the shape of heat transfer surface, the relative position, the temperature difference between surface and fluid, and the velocity of fluid, etc. Fig. 8 shows the distribution of convection heat transfer coefficient on the inner surface of the isolation sleeve (heat source surface) under different working conditions.

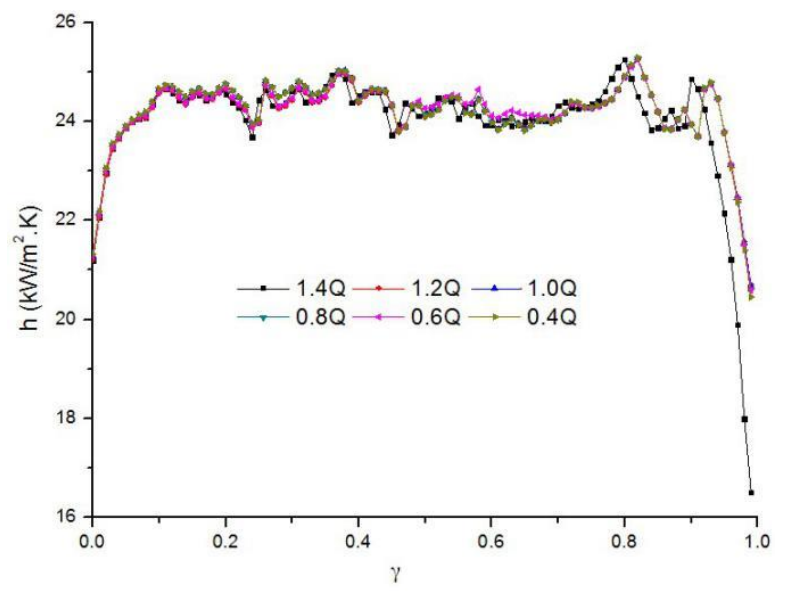

Fig. 8 the distribution of convection heat transfer coefficient on the inner surface of the isolation sleeve under different working conditions
The abscissa in the Fig. 8 represents the ratio of the length of the specified calculation point to the calculated length of the inner surface of the isolation sleeve, and the ordinate is the value $(\mathrm{h})$ of the convection heat transfer coefficient of the inner surface of the isolation sleeve (heat source surface) under different working conditions. It can be seen from Table 2 that under different working conditions, the eddy current loss and cooling cycle flow generated by the isolation sleeve are different, and the heat transferred and absorbed by the medium per unit volume is basically the same. As can be seen from Fig. 8, there is little difference in convection heat transfer coefficient of the inner surface of the isolation sleeve under different working conditions. The convection heat transfer coefficient on the inner surface of the isolation sleeve shows a slight wave peak change, and the overall change is not large. The convection heat transfer coefficient only changes greatly near the inlet and outlet close to the gap of the isolation sleeve on the inner surface of the isolation sleeve. The flow of medium near the outlet and inlet of the isolation sleeve clearance presents a state of sudden contraction and divergence, and the medium flow is disorder, which affects the convection heat transfer between the isolation sleeve and the medium. It can be seen from the comprehensive analysis that the magnetic drive coupling has a large convection heat transfer coefficient, a wide range of efficient convection heat transfer, and a good cooling performance of the cooling circulating channel.

\section{2. 2. The temperature and pressure distribution under the design conditions}

Three monitoring lines are set at different positions within the gap of the isolation sleeve. The head and tail of the monitoring line are in line with the head and tail of the gap of the isolation sleeve. The distance between the monitoring line and the inner wall (heat source surface) of the 
isolation sleeve is $0.0,0.4$ and 0.8 times the width of the gap of the isolation sleeve, respectively. Fig. 9 shows the pressure and temperature distribution at different positions within the gap of the isolation sleeve under the design condition.

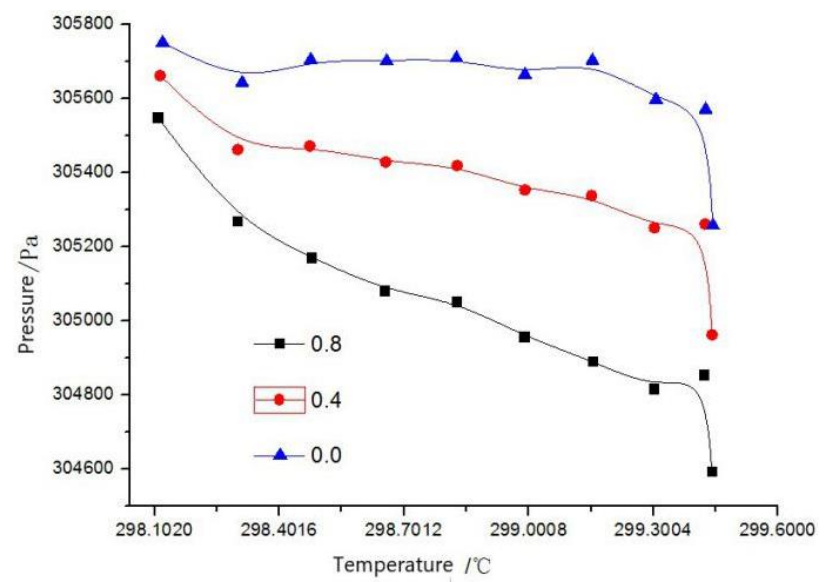

Fig. 9 The temperature and pressure distribution at different positions in the isolation sleeve clearance under the design condition

As can be seen from Fig. 9, along the direction of the isolation sleeve clearance to the bottom of the isolation sleeve, the pressure in the isolation sleeve clearance gradually decreases and the temperature gradually increases. The closer to the inner wall (heat source surface) of the isolation sleeve, the higher the pressure and temperature within the gap of the isolation sleeve. In the direction away from the inner wall surface of the isolation sleeve, the pressure and temperature gradually decrease, which is basically consistent with the analysis in Fig. 4. In addition, it can be seen from Fig. 9 that the pressure value at any point in the gap of the isolation sleeve under the design condition is far greater than the corresponding vaporization pressure value at this temperature, and the maximum temperature rise is $1.4 \mathrm{k}$, so there is no cavitation phenomenon in the gap of the isolation sleeve under the design condition.

\section{3. 3. The temperature and pressure distribution under dif- ferent working conditions}

The temperature and pressure distribution in the gap of the isolation sleeve under different working conditions is shown in Fig. 10. It can be seen from Fig. 10 that the pressure distribution in the gap of the isolation sleeve is the largest under the condition of small flow rate. As the flow rate increases, the pressure in the gap of the isolation sleeve gradually decreases. The temperature change corresponding to each point in the gap of the isolation sleeve under different working conditions is very small, which is consistent with the analysis in Fig. 9. In addition, it can be seen from Fig. 10 that the pressure value at any point in the gap of the isolation sleeve under different working conditions is far greater than the corresponding vaporization pressure value at the temperature at that point, so cavitation phenomenon will not occur in the entire gap of the isolation sleeve.

\section{Experimental verification}

The external characteristic test of the magnetic drive pump was done with the open centrifugal pump test bench, and the test was carried out according to GB/T32162005《Rotodynamic pumps-Hydraulic performance acceptance tests-Grades 1, 2 and 3$\rangle$. The magnetic drive pump prototype and experimental equipment are shown in Fig. 11. The principle of the open centrifugal pump test bench is shown in Fig. 12.

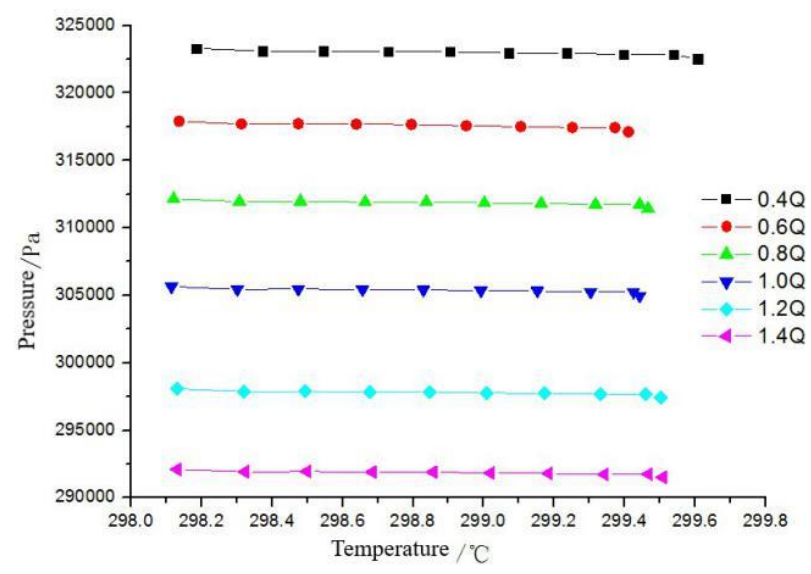

Fig. 10 The temperature and pressure distribution in the isolation sleeve clearance under different working conditions

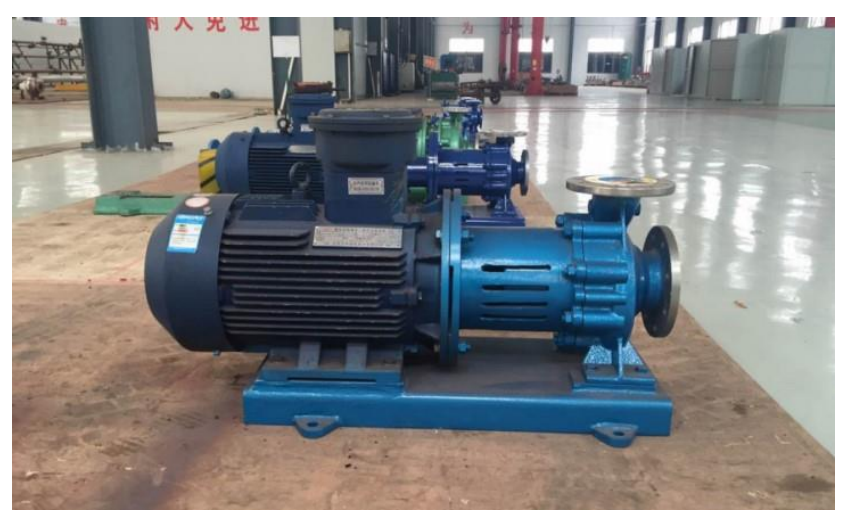

a

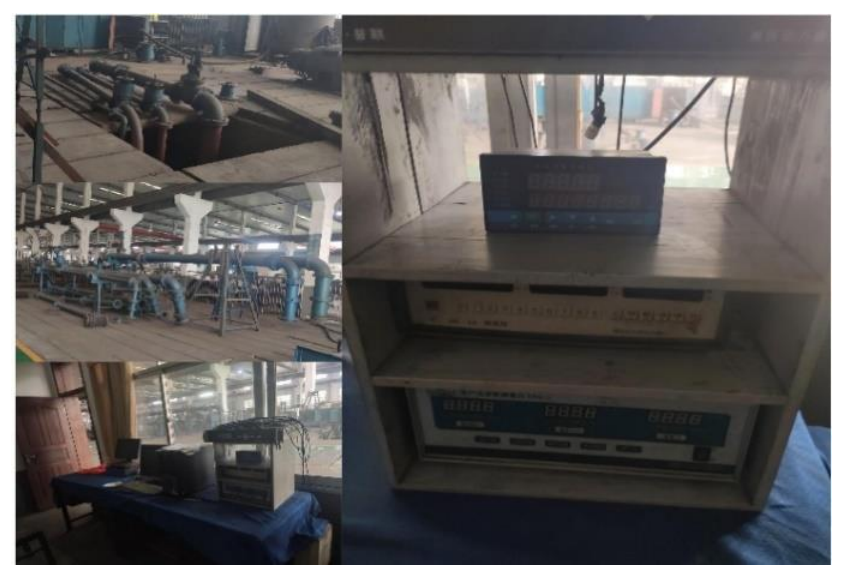

b

Fig. 11 The magnetic drive pump prototype and experimental equipment: a) Magnetic pump; b) Experimental device

This experimental device is equipped with valves, flow meters, flow meters, pressure sensors and other instruments. During the experiment, different working conditions were set, and the data under different working conditions were measured. The data needs preliminary processing to 
calculate the required parameters (efficiency, shaft power, head), and then visualize the parameters, and draw a graph for comparison.

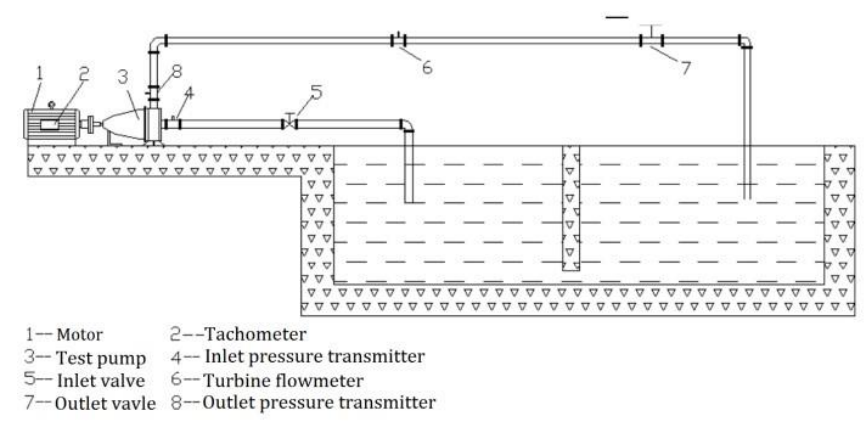

Fig. 12 The principle of open centrifugal pump test bench

The comparison between the calculation of the electromagnetic drive pump and the experimental results is shown in Fig. 13. As we can see from Fig. 13, The three sets of results of head, power and efficiency have the same changing trend. Among them, the numerical simulation results of power and the experimental results always tend to be consistent. Under standard conditions, the error of head is very small, and the size is only $1 \%$. While the efficiency has a more obvious error, reaching $6.8 \%$. After analysis, it is considered that the mechanical efficiency is not considered in the numerical calculation, so the numerical simulation result is greater than the experimental result. From the above analysis, as can be seen that the calculation results are in good agreement with the experimental results. The use of numerical calculation methods to study the flow-heat coupling of the cooling circuit of the magnetic drive centrifugal pump has a certain degree of reliability.

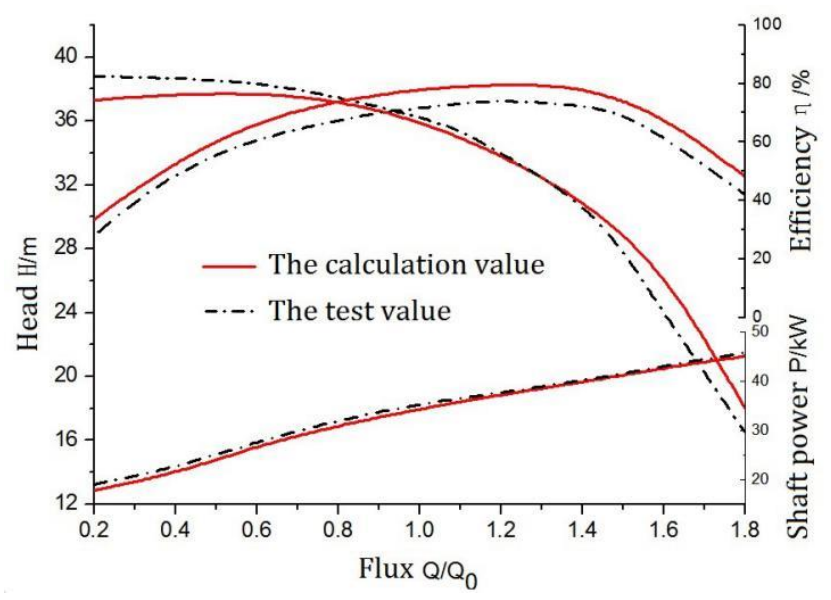

Fig. 13 The results of numerical calculation and the test on magnetic drive pump

\section{Conclusions}

1. The temperature of the isolation sleeve clearance in the cooling circulating channel is the highest near the bottom of the isolation sleeve. With the increase of the flow rate, the temperature of the medium at the bottom of the isolation sleeve and the reflow hole increase gradually. The temperature in the gap of the isolation sleeve gradually increases along the direction of the bottom of the isolation sleeve, and the highest temperature area appears near the bottom of the isolation sleeve and close to the wall surface. At the same longitudinal section, the temperature close to the wall surface in the gap of the isolation sleeve is higher than that far from the wall surface. The pressure in the gap of the isolation sleeve gradually decreases along the direction of the bottom of the isolation sleeve, and the lowest pressure area appears near the bottom of the isolation sleeve and far from the wall surface. According to the cavitation formation mechanism, under the same pressure condition, the higher the temperature, the more likely cavitation will occur. Therefore, cavitation is most likely to occur near the bottom of the isolation sleeve and close to the wall of the isolation sleeve.

2. The lowest pressure at the bottom of the isolation sleeve appears at the middle position at the bottom of the isolation sleeve. The maximum temperature area at the bottom of the isolation sleeve coincides with the minimum pressure area, and cavitation is easy to occur at this position. The temperature and pressure at the inlet of the reflow hole are relatively high. Along the exit direction of the reflow hole, the temperature and pressure inside the reflow hole gradually decreases, and the pressure in the area near the exit of the reflow hole is the lowest.

3 . The value of the convection heat transfer coefficient on the inner surface of the isolation sleeve has little difference under different working conditions. The peak fluctuation of convective heat transfer coefficient in most areas on the inner surface of the isolation sleeve is small. The convection heat transfer coefficient has a small change overall and only a large change takes place near the inlet and outlet of the inner surface of the isolation sleeve close to the gap of the isolation sleeve. The magnetic drive coupling has high convection heat transfer coefficient, the efficient range of convection heat transfer is wide and better cooling performance.

\section{Data availability and conflicts of interest}

The numerical data used to support the findings of this study are included within the article. The authors declare that they have no conflicts of interest.

\section{Acknowledgments}

This research was funded by the Opening Foundation of Engineering Research Center of Eco-environment in Three Gorges Reservoir Region, Ministry of Education (KF2019-04) the Open Research Subject of Key Laboratory of Fluid and Power Machinery (Xihua University), Ministry of Education grant number (szjj2017-098).

\section{References}

1. Thomas, S. K.; Muruganadam, T. M. 2018. A review of acoustic compressors and pumps from fluidics perspective, Sensors and Actuators A: Physical 283: 42-53. http://dx.doi.org/10.1016/j.sna.2018.09.031.

2. Brennan, J. R. 1999. Applications for magnetic drive rotary positive displacement pumps, World Pumps 1999(390): 24-28. http://dx.doi.org/10.1016/S0262-1762(00)87638-5.

3. Lugovaya, S.; Olshtynsky, P., Rudenko, A.; Tverdokhleb, I. 2012. Revisited designing of intermediate stage guide vane of centrifugal pump, Procedia Engineering 2012(39): 223-230. http://dx.doi.org/10.1016/j.proeng.2012.07.028. 
4. Tomski, L., Uzny, S. 2011. A hydraulic cylinder subjected to Euler's load in aspect of the stability and free vibrations taking into account discrete elastic elements, Archives of Civil and Mechanical Engineering 11(3):769-785. http://dx.doi.org/10.1016/S1644-9665(12)60115-0.

5. Su, H. S.; Chen, Z. P.; Zhao, M. Y.; Gao, Z. J.; Yu, W.; Zhang, Y. J. 2017. Characteristics of high-speed hydraulic coupler under axial vibration of pump wheel. Journal of agricultural engineering, 33(07): 51-57+315. http://dx.doi.org/10.11975/j.issn.10026819.2017.07.007.

6. Zhang, F.; Zhang, J. F.; Zhang, X. 2018. Internal flow characteristic analysis on outlet conduit based on whole flow field, Journal of agricultural engineering 36(12): 1246-1251.

http://dx.doi.org/10.3969/j.issn.1674-8530.18.0078.

7. Feng, J. S.; Liu, H. L.; Ding, J.; Wu, X. F.; Dong, L. 2017. Status of study on single channel centrifugal pumps and its developing tendency, Journal of agricultural engineering 35(3): 207-215. http://dx.doi.org/10.3969/j.issn.1674-8530.16.0018.

8. Kong, F. Y.; Zhou, S. Q.; Shen, X. K. 2011. Design of canned motor pump GPS40-9F, Journal of Drainage and Irrigation Machinery Engineering 29(1):16-20. http://dx.doi.org/10.3969/j.issn.1674-8530.2011.01.004.

9. Gamez-Montero, P. J.; Salazar, E.; Castilla, R.; Freire, J.; Khamashta, M.; Codina, E. 2009. Misalignment effects on the load capacity of a hydraulic cylinder, International Journal of Mechanical Sciences 51(2): 105-113. http://dx.doi.org/10.1016/j.ijmecsci.2009.01.001.

10. Kong, F. Y.; Chen K.; Yang, S. S. 2015. Design and development of experimental stand for pump as turbine, Journal of Drainage and Irrigation Machinery Engineering 33(5): 387-390. http://dx.doi.org/10.3969/j.issn.1674-8530.14.0153.

11. Amoabeng, K.O.; Choi, J.M. 2017. A study on the performance of a newly designed heat pump calorimeter, Applied Thermal Engineering 123: 216-225.

http://dx.doi.org/10.1016/j.applthermaleng.2017.05.029.

12. Baragetti, S.; Villa, S. 2016. Effects of geometrical clearances, supports friction, and wear rings on hydraulic actuators bending behavior, Mathematical Problems in Engineering 2016:1-17. http://dx.doi.org/10.1155/2016/3781397.

13. Leonzio, G. 2017. Mathematical model of absorption and hybrid heat pump, Chinese Journal of Chemical Engineering 25(10): 1492-1504. http://dx.doi.org/10.1016/j.cjche.2017.06.015.

14. Gao, Z. J.; Hong, F.; Liu, J. R.; Su, H. S. 2017. The impact of deflector hole diameters on the flow properties of magnetic drive pump, Journal of Engineering for Thermal Energy and Power 32(8): 78-88+150. http://dx.doi.org/ CNKI:SUN:RNWS.0.2017-08-014.

15. Tan, L.W.; Shi, W.D.; Kong, F. Y.; Zhang, D. S. 2015.
Design and numerical simulation of cooling circuit in magnetic pumps, Transactions of the Chinese Society of Agricultural Engineering (Transactions of the CSAE) 31(8): 84-91.

http://dx.doi.org/10.3969/j.issn.1002-6819.2015.07.013.

16. Blázquez, C. S.; Borge-Diez, D.; Nieto, I. M.; Martín,

A. F.; González-Aguilera, D. 2019. Technical optimization of the energy supply in geothermal heat pumps, Geothermics 81: 133-142.

http://dx.doi.org/10.1016/j.geothermics.2019.04.008.

17. Gamez-Montero, P.J.; Salazar, E.; Castilla, R.; Freire, J.; Khamashta, M.; Codina, E. 2008. Friction effects on the load capacity of a column and a hydraulic cylinder, International Journal of Mechanical Sciences 51:145-151. http://dx.doi.org/10.1016/j.ijmecsci.2008.12.007.

18. Wang, Y.; Liu, H. L.; Liu, D. X. et al. 2013. Effects of vane wrap angle on flow induced vibration and noise of centrifugal pumps, Transactions of the Chinese Society of Agricultural Engineering (Transactions of the CSAE) 29(1): 72-77.

http://dx.doi.org/10.3969/j.issn.1002-6819.2013.1.010.

Zh. Gao, J. Zhang, W. Li, Ch. Hu, Ch. Si, F. Hong, J Liu, X. Shi

\section{THE FLOW-HEAT COUPLING STUDY OF COOLING CIRCULATING CHANNEL OF MAGNETIC DRIVE PUMP}

S u m m a r y

The medium in the cooling circulation channel of the magnetic drive centrifugal pump will take away the heat generated by the magnetic eddy current and bearing friction in time to avoid the high temperature demagnetization phenomenon of the permanent magnets. Therefore, the reasonable design of the cooling circulation channel directly affects the stable operation of the magnetic drive centrifugal pump. In this paper, the heat exchange and temperature distribution in the cooling circulating channel of magnetic drive pump are studied by means of numerical calculation of fluid-heat coupling and external characteristic test. The temperature distribution and development law of the isolation sleeve clearance, the bottom of the isolation sleeve and the reflow hole are analysed emphatically, and the convection heat transfer coefficient distribution in the isolation sleeve clearance is studied.

Keywords: magnetic drive centrifugal pump, cooling circuit channel, flow-heat coupling, convection heat transfer, numerical calculation.

Received November 16, 2020 Accepted August 07, 2021

This article is an Open Access article distributed under the terms and conditions of the Creative Commons Attribution 4.0 (CC BY 4.0) License (http://creativecommons.org/licenses/by/4.0/). 